\title{
What Is the Evidence Supporting the Use of Surgical Face Masks in the Operating Room in Preventing Surgical Site Infection after Clean Surgery? A Systematic Review
}

\author{
Priscilla 0. Aikabeli \\ Department of Nursing Science, Benson Idahosa University, Benin City, Nigeria \\ Email: emikeaikabeli@yahoo.com
}

How to cite this paper: Aikabeli, P.O. (2021) What Is the Evidence Supporting the Use of Surgical Face Masks in the Operating Room in Preventing Surgical Site Infection after Clean Surgery? A Systematic Review. Open Journal of Nursing, 11, 847-870.

https://doi.org/10.4236/ojn.2021.1110070

Received: April 26, 2021

Accepted: October 18, 2021

Published: October 21, 2021

Copyright $\odot 2021$ by author(s) and Scientific Research Publishing Inc. This work is licensed under the Creative Commons Attribution International License (CC BY 4.0).

http://creativecommons.org/licenses/by/4.0/

\begin{abstract}
Background: Facemask is an essential component of the surgical outfit adorned by operating room staff to filter microorganisms by droplets from the oral and nasopharynx of the personnel, thereby reducing contamination, protecting the patient's wound and minimising the risk of Surgical Site Infections (SSI). Objective: The objective of this review was to explore the available evidence and provide a better understanding of the effect of a surgical facemask in preventing SSI in clean surgery performed in the operating room. Data sources: Key electronic databases related to nursing, allied health, life science, biomedicine and research were searched for published literature on the use of facemask in the operating room. Methodology: A systematic review of quantitative research studies of randomised controlled trials was conducted with a meta-analysis of the results. Results: No variation in the rate of infection between the two (masked and unmasked) groups. Conclusion: The effect of facemask in minimising SSI after clean surgery remains questionable due to the limited results. More comprehensive research is needed.
\end{abstract}

\section{Keywords}

Operating Room, Operating Theatre, Facemask, Clean Surgery, Surgical Site Infection

\section{Introduction}

This study is a systematic review, carried out to critically appraise and synthesise the existing knowledge on the effectiveness of the use of surgical facemasks in 
the operating room in preventing surgical site infection (SSI) in clean surgery. The aim of the study is focused on the theoretical framework of clinical decision-making for evidence-based practice [1]. The review identified gaps in knowledge as there is paucity of data and evidence of randomised controlled trials (RCT) on this topic in the available literature [2] [3]. The review also highlighted inappropriate use and inconsistency in facemask practice by operating room personnel which could lead to surgical wound contamination, subsequent wound infection and the attendant increase in care cost, longer hospitalisation and mortality [2]. These and other areas of doubt were refined into a specific researchable question [4].

This systematic review will provide a standpoint to underpin evidence-based decision-making with a focus on the research question, "What is the evidence supporting the use of surgical face masks in the operating room in preventing surgical site infection after clean surgery?" [4]. Additionally, this review evaluated the strengths and weaknesses of the evidence-based practice of facemask use in the operating room in preventing SSI.

\section{Why This Study Is Necessary}

The rationale for this current review, therefore, is to provide knowledge of best practices to prevent SSI. Consequently, to evaluate the current evidence on the effect of facemask use to inform practice decision-making which will help to improve current clinical practice and enhance their appropriate use to minimise SSI rate, reduce care cost, length of hospitalisation and mortality rate. The outcome of this review will be published to educate surgical team members and enhance knowledge improvement in the practice of facemask use in the operating room to minimise SSI.

\section{Background}

The use of surgical facemasks in the operating room to minimise contamination has been standard practice for over a century [5] [6]. They were originally invented by Paul Berger in 1897 to protect patients from the risk of SSI by filtering microorganisms from droplets exhaled from the nose and mouth of the surgical team during surgery [7]. More recently, in the wake of blood-borne infections, facemask use is advocated in Standard Precautions as part of Personal Protective Equipment to protect the surgical team from the patient [2] [8] [9]. However, this review did not include the use of facemask for this purpose.

The European Centre for Disease Prevention and Control [10] acknowledge that Hospital Acquired Infection (HAI) is currently a significant Public Health challenge. They highlighted that SSI is the $3^{\text {rd }}$ commonest, accounting for $13 \%$ 17\% of HAIs in Europe. Similarly, in England, Plowman, Graves and Griffin [11] highlighted that the socio-economic burden of contracting SSI can be estimated in longer hospitalisation and increased care cost. The Office of National Statistics [12] also emphasised that when juxtaposed with all acute care hospitals, the 
annual cost is almost $£ 1$ Billion to the NHS with the attendant morbidity and mortality. Additionally, Spruce [7] agreed that the direct cost annually to the US healthcare system is between $\$ 28.4$ and $\$ 45$ billion. Correctly worn, facemask covers the nose and mouth, metal band curved on the bridge of the nose and tapes tied round the back of the head [13]. Although facemask was primarily designed to protect the patient, Vincent and Edwards [3] highlighted several ways in which incorrect use could result in surgical wound contamination as illustrated below Figure 1.

\subsection{Problem Statement}

Evidence from studies [2] [3] [13] show that the practice of facemask use is inconsistent and highlight an existing controversy over their clinical effectiveness. However, organisations with operating department concern [8] [14] [15] [16] currently recommend the practice. From the review of available literature [2] [3] [8], this researcher concluded that on the one hand, the practice is part of the long standing tradition of procedures and practices of maintaining theatre discipline presumed to minimise the transfer of bacteria by creating a barrier between the nose and mouth of operating room personnel and an open patient wound.

On the other hand, facemask may be protective only when used appropriately. Additionally, as a trained perioperative nurse, this researcher's experience and observation in the operating rooms of public hospitals in Nigeria showed that some surgical team members are consistent in wearing facemask appropriately while some either wear incorrectly or do not wear at all. By deciding not to wear a mask, the patient may be left vulnerable to the risk of wound infection by droplet contamination [3] after clean surgery. The search did not identify any

- A way of carrying liquid by capillary action and causing possible bacterial passage.
Wicking
Wigling
of the face.
Incorrect
wearing Exposing the nose or mouth.
Incorrect
disposal By grasping the filter end and contaminating the wearer's hands.

Figure 1. Incorrect ways of using surgical face mask. 
literature on the effectiveness of facemask use in preventing SSI in clean surgery performed in the operating room.

There is evidence to question the use of facemasks as there is no adequate rationale for their continued use in the operating room. Furthermore, the search revealed a number of literature analysing outcome measures, including primary research using disparate methodologies and providing mixed results [17]. The above discussion shows the effectiveness of facemask use in the operating room in preventing SSI is inconsistent. This paucity and inconsistency of evidence provided the rationale for the systematic review to analyse and synthesise available research. From the findings above, this researcher was prompted to question the effectiveness of facemask use during clean surgery in the operating room to prevent SSI. Therefore, it presented the need for a systematic review to consolidate current knowledge, update the evidence-base, direct future research and inform decision-making on the practice of facemask by enhancing their appropriate use in preventing SSI which was imperative. It is intended to add to the body of knowledge of what is already known, influence policy decision-making and enhance appropriate use and education on safe evidence-based practice.

\subsection{Research Question}

As expounded above, the research question is: "What is the evidence supporting the use of surgical facemasks in the operating room in preventing surgical site infection after clean surgery?"

\subsection{Aim of the Review}

The aim of this review is to establish current evidence on the effect of facemask use in the operating room in preventing SSI and its resultant effect of increased care cost, longer hospitalisation and mortality rate.

Provide evidence to enhance appropriate use to improve practice and inform decision-making in reducing SSI rate.

To add to the body of knowledge on what is already known on the effect of facemask use in the operating room.

\subsection{Objective}

To explore available literature and determine whether or not facemask use in the operating room during clean surgery minimises SSI, reduces care costs, length of hospitalisation, morbidity and mortality rate and enhance appropriate use.

Formulate recommendations for training and highlight areas for further research.

\section{Methodology and Protocol}

\subsection{Research Design}

This systematic review critically appraised the available evidence in answering the research question as systematic reviews aim at identification, selection, ap- 
praisal and synthesis of primary research findings [18]. Systematic reviews provide unbiased assessment of substantial evidence to identify gaps in knowledge and highlight reasons for future research where necessary [19] and ultimately inform policy decision-making in healthcare practice and improve patient outcomes. Systematic reviews stem from the positivist tradition, a paradigm defined by Weaver and Olson [20] as a school of thought with set beliefs, each having separate ontology, epistemology and methodology presenting varying degrees of knowledge.

Weaver and Olson [20] described the positivist paradigm as highly scientific, believing that human and social occurrences can be viewed by scientifically generating and testing a hypothesis with quantitative methodology and experimental design. This is followed by meta-analysis to demonstrate the veracity and produce up to date information to validate the hypothesis as a process of deductive theory generation. As a result, the world can reliably be described with an objectively generalizable theory which has been developed from an epistemological point of view. Furthermore, this is independent of the thoughts and experiences of the population, known in positivist paradigm as the ontological perspective [21].

Therefore, this study embraced the positivist paradigm in systematic generation, appraisal and understanding of quantitative research data. This study is a quantitative, explorative, descriptive design, traditionally used in addressing research questions in systematic reviews [22]. Although Bunniss and Kelly [21] advice that no specific research design is considered more valuable than the other, they suggest that the best design is the one that most appropriately and objectively addresses the research question. This design was best suited as only quantitative research studies with RCT on the effect of facemask use in clean surgery in the operating room met the inclusion criteria for this review to objectively answer the research question with a research protocol as discussed in the next section.

\subsection{Research Protocol}

The first part of a standard systematic review is to define the research question [18] using a research protocol to plan clear sections and set boundaries for the review. As already stated, the research question is: "What is the evidence supporting the use of surgical face masks in the operating room in preventing surgical site infection after clean surgery?" The PICOS framework was utilised to define the research question as Bettany-Saltikov [18] stated that PICOS framework is frequently used to develop quantitative research questions. The elements of this framework are presented in Table 1.

\subsection{Search Strategy}

The review was undertaken to identify and critique available relevant literature and provide an overview of what is currently known, evaluate the evidence supporting the use of facemasks in preventing SSI and validate the research question 
Table 1. PICOS framework.

\begin{tabular}{ll}
\hline Population & All patients undergoing clean surgery in the operating room \\
Intervention & Use of surgical face mask \\
Comparator & Non-use of face mask \\
Outcome & Incidence of surgical site infection, cost, length of hospital stay, mortality rate \\
Study Design & Systematic reviews/meta-analysis, Randomised Controlled Trials
\end{tabular}

[23]. The literature search aided the investigation of the objective and established the evidence which underpinned the review [23]. To identify studies relevant to the research, an initial Internet search was undertaken. No limits were used at this stage as a general survey of available literature pertinent to the topic under review needed to be scoped and appraised. Most of these articles were not available on full text, a necessary tool in systematic reviews [24].

The foundation and structure for identifying keywords used for searching electronic databases in finding articles relevant to the review is the research question. To refine the search and connect it to the research question, a more advanced search with the keywords "surgical face masks", "operating room", "operating theatre", "surgical site infection" and the limits "clean surgery" and "review" was undertaken in BNI, MEDLINE, CINAHL and the Cochrane Database for Systematic Reviews. These databases were chosen for their specialties and acclaim in life science, biomedicine, nursing, allied health professional journal articles and review. Filters were then applied to studies published from 2001 to obtain more recent articles. Boolean operators and truncations were then applied with the filters English language to ensure a comprehensive search as this researcher is only proficient in English language. An adequate record of the search and databases used are illustrated in a table (Appendix 1) to maintain the transparency and replicability of the search strategy [25], a significant component of a quality systematic review.

Consideration was also given to methodological approach to ensure the review is balanced [18]. A manual search was conducted to examine bibliography and reference list of journal articles, grey literature like unpublished theses to obtain relevant articles which may have been omitted in the previous search to confirm the inclusion of all data relevant to the review [23]. However, no unpublished literature was identified to have met the inclusion criteria. Effort was made to contact the authors but there was no response. WHO, NICE and AORN guidelines were accessed as they are relevant to the topic under review. Synthesis was by quality, design and heterogeneity of the identified studies.

To ensure the truthfulness and validity of the search results, Jolley [19] stressed the importance of documentation of the process of decision-making in selection of studies for systematic reviews which can be validated by others. Therefore, the results of the search for this systematic review and the reasons for exclusion of studies are clearly illustrated in the diagram of the PRISMA flow chart below Figure 2 . 

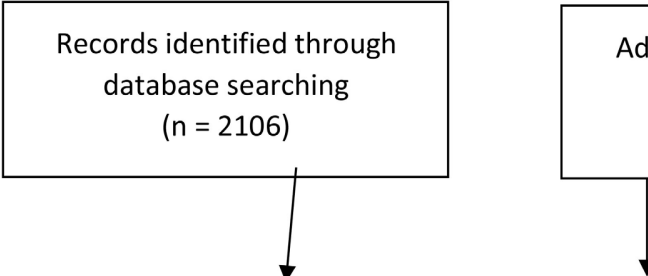

Additional records identified through other sources

$$
(n=0)
$$
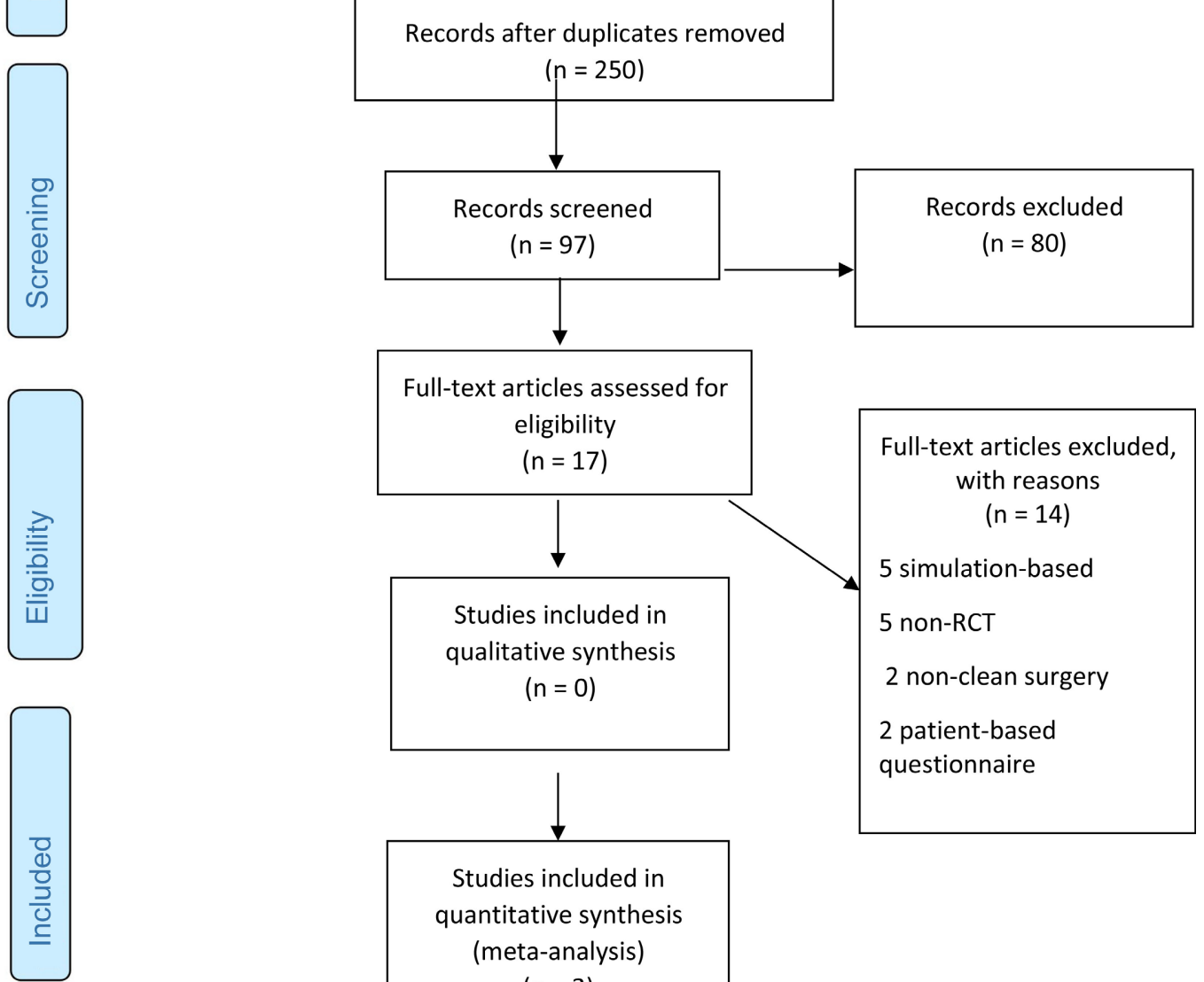

Studies included in quantitative synthesis (meta-analysis)

$$
(n=3)
$$

From: Moher D, Liberati A, Tetzlaff J, Altman DG, The PRISMA Group (2009). Preferred Reporting /tems for Systematic Reviews and Meta-Analyses: The PRISMA Statement. PLoS Med 6(6): e1000097. doi:10.1371/journal.pmed1000097

For more information, visit www.prisma-statement.org.

Figure 2. PRISMA flow chart.

\section{Study Selection}

The standard selection criteria for inclusion and exclusion were followed to establish the relevance of all the studies identified by the search strategy. All articles obtained had an initial assessment of their titles and abstracts to ascertain those that fully met the inclusion criteria. Developing the research question utilising the PICOS framework helped in formulating the criteria for study selection [18] as presented in Table 2. 
Table 2. Selection criteria.

\begin{tabular}{|c|c|c|}
\hline PICOS & Inclusion Criteria & Exclusion Criteria \\
\hline Population & $\begin{array}{l}\text { All patients undergoing clean } \\
\text { surgery in the operating room }\end{array}$ & $\begin{array}{l}\text { Non-clean surgery, procedures not } \\
\text { performed in the operating room, } \\
\text { simulation based without actual surgery }\end{array}$ \\
\hline Intervention & Use of disposable face mask & Type of face mask not specified \\
\hline Comparator & No face mask & $\begin{array}{l}\text { No restriction of non-scrubbed } \\
\text { staff from comparator group }\end{array}$ \\
\hline Outcome & $\begin{array}{l}\text { Wound infection as defined by } \\
\text { guidelines for wound classification }\end{array}$ & Follow up time not specified \\
\hline Types of study & $\begin{array}{l}\text { All research published in peer } \\
\text { reviewed journals, RCT, full text } \\
\text { English language } \\
\text { From 2001-2016 }\end{array}$ & $\begin{array}{l}\text { All studies published before } 2001 \text {, } \\
\text { non-English studies, non RCT, patient } \\
\text { based questionnaire, } \\
\text { Duplicate studies } \\
\text { No full text }\end{array}$ \\
\hline
\end{tabular}

Where it was not clear from screening the titles and abstracts, a full text copy of the article was obtained. In comparison, Birgand et al. [17] and Vincent and Edwards [3] demonstrated similarity in the process of selection through a number of stages where the review authors jointly compared the studies before deciding whether or not they met the inclusion criteria. Furthermore, Vincent and Edwards [3] highlighted that titles and abstracts of articles identified by the search strategy were assessed by one review author and where there was disparity, full text was obtained by another review author. They also stated that two review authors jointly made decision on which studies met the inclusion criteria and discussed all decisions with a member of the Cochrane Wounds editorial board.

It is agreed by Bryman [23] and Bettany-Saltikov [18] that a quality systematic review involves two or more review authors in making a consensus decision in the selection of studies for a review. However, as only one researcher undertook this systematic review, the selection and decision making process of study inclusion and exclusion were unilateral, which is a limitation of the study. Nonetheless, a research protocol was utilised to clearly demonstrate the process of selection. Not only did this research protocol show the clarity of purpose of the review, but also, it ensured that the plan was not deviated from.

Copies of all the studies that fully met the selection criteria were obtained and the quality assessed to systematically highlight the strengths and weaknesses [24]. The assessment process was used to appraise how much the research question was answered by the study design, method, analysis and presentation and eliminated bias, an internal validity where bias can be due to chance or the study methodology [19]. The usefulness of the study to surgical practice settings and the trustworthiness of the findings were appraised in the assessment process. Due consideration was also given to the relevance of the research to surgical practice and the generalizability of the findings to all categories of the surgical 
patient population and clean surgery in the operating room to confirm the external validity of the research [19]. There are appraisal tools for this process. An example of these tools is the CASP which considers the validity of the study and the application of its results to local health settings. CASP was utilised in this study as it embraces the appraisal of quantitative methods and RCTs in deciding the quality of the study [24].

\subsection{Summary of Critical Appraisal}

The quality of this systematic review is limited by the size and number of studies included and shows there is paucity of information on the topic. Two RCTs with unclear allocation concealment were included in the study by Birgand et al. [17] systematic review and meta-analysis of the effect of surgical facemasks in minimising the rate of SSI in clean surgeries. Vincent and Edwards [3] had no allocation concealment and Webster et al. [26] had true allocation concealment. All studies presented analysis of risk of bias, quantified conclusions and provided the statistical power of all studies included and described literature search strategy with predetermined exclusion criteria. None of the studies pooled data from the trials included. Above all, documentation of decisions and findings were completed and presented to ensure a transparent rigour process as shown in $\mathrm{Ta}$ ble 3 below.

Table 3. Rigour process: critical appraisal.

\begin{tabular}{|c|c|}
\hline Articles Included & RCTs $(\mathrm{n}=3)$ \\
\hline \multicolumn{2}{|l|}{ Study 1: [26] } \\
\hline Methods & Randomised Controlled Trials \\
\hline \multirow[t]{4}{*}{ Participants } & All patients undergoing clean surgery - 660 patients. \\
\hline & Inclusion criteria: not stated. \\
\hline & Exclusion criteria: existing airborne infection where face mask was specifically required. \\
\hline & Participants had baseline similarity for age, gender, weight, prophylactic antibiotics and ASA classification. \\
\hline Interventions & Group 1: Mask $(\mathrm{n}=313)$ \\
\hline Comparator & Group 2: No mask $(\mathrm{n}=340)$ \\
\hline \multirow[t]{3}{*}{ Outcomes } & $\begin{array}{l}\text { National Nosocomial Infection Surveillance System definition of wound infection used: superficial, deep } \\
\text { and organ space. }\end{array}$ \\
\hline & Group 1: Mean follow up 33.4 days (SD 22.1) \\
\hline & Group 2: Mean follow up 33.4 days (SD 22.8) \\
\hline \multirow[t]{2}{*}{ Comments } & Missing data for 7 clean cases created a unit of analysis error. \\
\hline & $\begin{array}{l}\text { Only non-scrubbed staff was asked to comply with randomisation. Scrubbed staff was not included in the } \\
\text { trial. }\end{array}$ \\
\hline Risk of Bias & Computer-generated randomisation of operation lists into two groups, mask and no mask and allocation \\
\hline Selection bias (low risk) & $\begin{array}{l}\text { was by phone call immediately before the session commenced, to a person who was unaware of the type of } \\
\text { list in the theatre reducing the risk of selection bias. }\end{array}$ \\
\hline $\begin{array}{l}\text { Blinding performance and detection } \\
\text { bias (low risk) }\end{array}$ & $\begin{array}{l}\text { Patients unaware of allocation. Data of any post-operative wound infection was obtained by routine } \\
\text { surveillance of infection control staff that was unaware of the treatment protocol. }\end{array}$ \\
\hline Baseline comparability of treatment & The two groups were comparable for baseline characteristics of type of surgery, wound and ASA \\
\hline and control (low risk) & classification as well as age, gender, pro-operative hospitalisation, BMI and prophylactic antibiotics. \\
\hline Conflict of Interest (unclear risk) & Source of funding not stated. \\
\hline
\end{tabular}

Article 2: [17] 


\section{Continued}

Methods

Participants

\section{Interventions}

Comparator

Outcomes

Comments

\section{Risk of Bias}

Random sequence/Allocation concealment (selection bias high-risk) Blinding (performance and detection bias)

Baseline comparability of treatment
Randomised Controlled Trials.

1429 clean surgery performed in the operating room.

Inclusion criteria: surgical incision through intact skin with primary wound closure.

Exclusion criteria: patients not informed or consent not given for all category of surgery.

Baseline comparability: similar for age, acute and cold surgery.

Group 1: Mask $(\mathrm{n}=706)$.

Group 2: No mask $(n=723)$.

Wound infection defined by visible pus and/or cellulitis requiring debridement, drainage with antibiotic treatment.

Follow up was until after discharge but no post discharge findings stated.

Patients had 2 to 3 body washes pre-operatively with $4 \%$ Chlorhexidine before elective surgery, at least 1 body wash in most acute cases, creating a unit of analysis error.

A random list was set up for 1 year, denoting weeks as "masked" or "unmasked". The list was inversed for the $2^{\text {nd }}$ and $3^{\text {rd }}$ part of the year to avoid seasonal differences between the groups, making selection bias a high risk. Concealment inadequate as investigators enrolling participants could possibly foresee allocation and thus introduce selection bias.

Patient blinding not described (unclear bias).

Notification of the trial was issued with each wound swab (high risk).

Baseline comparability stated for age and type of surgery, wound, ASA classification, preoperative

hospitalisation, BMI and prophylactic antibiotics.

Study 3: [3]

Methods

Participants

Interventions

Comparator

Outcome

Comments
Randomised controlled trials

2113 patients undergoing clean surgery in the operating room.

Inclusion criteria: clean surgery in the operating room.

Exclusion criteria: unclean surgery not done in operating room.

Group 1: Mask $(\mathrm{n}=1036)$.

Group 2: No mask $(\mathrm{n}=1077)$.

Wound infection without standard follow up time of included studies.

Limited by size and number of included studies. Publication bias could not be assessed due to the small number of studies included. Heterogeneity of included studies prevented pooling of data and meta-analysis.

Risk of Bias

\subsection{Ethical Appraisal}

Assessment and appraisal of the ethical qualities of the studies that met the inclusion criteria for the review was undertaken to meet the guidelines for research ethics as outlined by the National Institutes of Health Organisations for biomedical research involving human subjects [27]. The principles of these ethical guidelines include beneficence, non-maleficence, justice, fidelity, veracity, confidentiality and the application is relevant for all types of research with varying populations and settings [28]. The AGREE11 (Appraisal of Guidelines for Research and Evaluation) tool developed for quality appraisal guidelines in health and social care research [29] was also used.

These ethical principles were considered as operating room personnel and patients undergoing clean surgery were included in the population, with any other information regarding facemask use in the operating room which researchers need to know. These considerations include the self-conduct of researchers and the way they treat participants during the process of the research [23]. David and Resnik [28] include goal, duty of care, rights of participants and global consider- 
ations as guide for the process of determination of the ethical quality of each study before inclusion in a systematic review. Birgand et al. [17] and Vincent and Edwards [3] showed application of this guide as they highlighted that ethical approval was sought and obtained before conducting their studies.

Although Webster et al. [20] and Vincent and Edwards [3] stated that they had both internal and external funding; however, the authors of the three included studies stated no conflict of interests. In addition, they highlighted that patients were told they could exercise their right of withdrawal from the study at will, strengthening their readiness and right of participation in the study [30]. Additionally, all three studies ensured confidentiality by maintaining anonymity of all participants. Birgand et al. [17] and Vincent and Edwards [3] stated that they obtained informed consent from the operating room staff who participated in the studies. Furthermore, Birgand et al. [17] highlighted they obtained informed consent from patients. In addition to obtaining informed consent from patients and personnel, Vincent and Edwards [3] specified that they gave forms to all participants and told them to contact the researcher and express their willingness to participate in the study. These efforts clearly illustrate the researchers considered guidelines for research ethics and maintained ethical standards in their conduct and behaviour as they protected the rights of all the participants involved in the research [27] [28].

As this study was a systematic review, no direct human participants were involved. Therefore, no consent was required. However, this researcher undertook the Epigeum course on Good Research Ethics practice.

Two vital aspects of a systematic review, first evaluation of rigour and second, appraisal of ethical quality of all studies selected for inclusion in the systematic review have been explored as advised by Petticrew and Roberts [4]. The eventual quality of the systematic review is dependent upon the final quality, selection and appraisal of the data extracted from the studies included in the review [31].

\subsection{Data Extraction}

Data extraction is the process of retrieving vital information concerning the characteristics and findings from the studies that met the inclusion criteria that are relevant in answering the research question, as they relate to the effectiveness of surgical facemasks in reducing SSI rate. Studies not RCTs were excluded from this systematic review. The following information were extracted from each of the included studies-author, title, type of study, types of surgery, trial setting, number of wound infections, co-interventions, remarks, opinions or limitations, implications for practice and any other comments identified in the studies. Jni, Altman and Egger [32] recommend and Fain [33] agrees proper data extraction and documentation as a bench mark of the quality of the review process to enhance the validity of the results. Therefore, extraction and summary of details of identified research studies was done with the aid of a piloted data extraction form as presented in Table 4 below. 
Table 4. Data extraction summary.

\begin{tabular}{|c|c|c|c|c|}
\hline Author, title and type of study & $\begin{array}{l}\text { Population and } \\
\text { type of surgery }\end{array}$ & $\begin{array}{l}\text { Trial setting and } \\
\text { co-interventions }\end{array}$ & $\begin{array}{c}\text { Outcome measures, } \\
\text { no. of wound infections }\end{array}$ & $\begin{array}{l}\text { Comments, opinions } \\
\text { and limitations }\end{array}$ \\
\hline Study 1 [26] (Primary research) & $\begin{array}{c}660 \text { patients undergoing } \\
\text { clean surgery }\end{array}$ & $\begin{array}{c}\text { Operating room. } \\
\text { Mask versus No mask }\end{array}$ & $\begin{array}{l}\text { Wound infection. } \\
\text { No. not specified. }\end{array}$ & $\begin{array}{c}\text { Scrubbed staff } \\
\text { not included in the trial. }\end{array}$ \\
\hline $\begin{array}{c}\text { Study } 2 \text { [17] } \\
\text { (Systematic review) }\end{array}$ & $\begin{array}{l}1429 \text { undergoing } \\
\text { elective surgery }\end{array}$ & $\begin{array}{c}\text { Operating room. } \\
\text { Mask versus No mask }\end{array}$ & $\begin{array}{l}\text { Wound infection. } \\
\text { No. not specified. }\end{array}$ & $\begin{array}{l}\text { Unit of analysis } \\
\text { error present }\end{array}$ \\
\hline Study 3 [3] (Systematic review) & $\begin{array}{l}2113 \text { patients undergoing } \\
\text { clean surgery }\end{array}$ & $\begin{array}{c}\text { Operating room. } \\
\text { Mask versus No mask }\end{array}$ & $\begin{array}{l}\text { Wound infection. } \\
\text { No. not specified. }\end{array}$ & $\begin{array}{l}\text { Unit of analysis } \\
\text { error present }\end{array}$ \\
\hline
\end{tabular}

\subsection{Data Analysis and Synthesis}

Meta-analysis and synthesis was undertaken in answering the research question as the review detailed data extraction of quantitative research articles. As earlier mentioned, the process of data synthesis was determined by the quality, design and heterogeneity of the research articles identified. The notable findings and themes that emerged were analysed and compared to establish the similarities and differences. Areas for further comprehensive research were identified as the authors all recommended further robust research on the topic to enhance evidence-based clinical practice and improve patient outcomes. In addition, the findings could be entered into the Cochrane Review Manager [34] as done by Vincent and Edwards [3] to ensure the findings are reliable. Details of the data analysis and synthesis (results) are presented in a table.

\section{Results}

The initial search produced 2106 hits. The advanced search revealed 250 hits out of which 97 were retrieved for more scrutiny. After reading the abstracts, 80 were irrelevant, 17 had potential but 14 were excluded after reading the titles, abstracts and full text for their relevance to the research question and most recent year of publication. 3 studies were included in the review; Webster et al. [26], Birgand et al. [17] and Vincent and Edwards [3] based on the selection criteria as previously shown in Figure 2 above. A summary of the search results is tabulated below Table 5 .

\subsection{Characteristics of Included Studies}

The search disclosed numerous reviews on the use of surgical facemasks in the operating room to recognise any existing narrative or systematic reviews on the topic to establish the originality of the research question [18] [25]. All the reviews on the topic have similarities and differences to the research question. One of these reviews [3] concentrated on clean surgeries performed in the operating room which is the focus of the research question. Webster et al. [26], a primary research and Birgand et al. [17] incorporated clean, unclean or contaminated surgeries performed in the operating room. However, data extracted for this review were for clean surgery only. 
Table 5. Summary of search results.

\begin{tabular}{cccc}
\hline Database & Initial Search & Full Text & Appraisal \\
\hline Google Scholar & 32 & 3 & 0 \\
BNI & 26 & 0 & 0 \\
MEDLINE & 1600 & 1 & 0 \\
CINAHL & 410 & 9 & 1 \\
Cochrane & 38 & 4 & 2 \\
Total & 2106 & 17 & 3 \\
\hline
\end{tabular}

\subsection{Summary of Evidence}

The evaluation of the summary of the evidence from the 3 RCTs and meta-analysis on the effectiveness of facemask use in the operating room in reducing SSI rate revealed limited inconclusive results whether or not the use of facemask has any effect on wound infection in clean surgery. The strength of evidence from 2 of the studies was strong and 1 study was weak. Comparison was made between masked and unmasked groups of the surgical team in reducing bacterial contamination of an open patient wound by operating room personnel during clean surgery. Findings revealed the same unclear results of the clinical effectiveness of facemask use in the operating room in preventing SSI. A summary of the characteristics of these studies is presented in Table 6 below.

\subsection{Characteristics of Excluded Studies}

A total of 14 studies were excluded. 5 studies were theatre based simulation which did not involve actual surgery [35] [36] [37] [38] [39]. In all these studies, outcome measure for contamination was by the use of settle plates. 2 were not clean surgery [40] [41] and the studies were discontinued due to the high level of contamination on the settle plates and postoperative wound infections discovered. 2 studies [42] [43] assessed SSI with the use of patient questionnaire. 5 were not RCTs [13] [43] [44] [45] [46]. These studies excluded at full text reading with reasons for exclusion are presented in a table as Appendix 2.

\subsection{Effect of Intervention}

All studies included in the review compared face mask use with non-use in 4202 patients undergoing clean surgery in the operating room. Homogeneity was assessed clinically and methodologically. The clinical heterogeneity observed between the trials was measured by study population, diagnosis of SSI and duration of follow up. Vincent and Edwards [3] highlighted that this could have been influenced potentially by the type of face mask, restriction of non-scrubbed personnel from the intervention group, air flow and design of the operating theatre and place of study. These factors made it difficult to pool the studies.

\subsection{Outcome Measures Used in the Included Studies}

Mangram et al. [47] and Anderson [48] argued about the most credible outcome 
Table 6. Characteristics of included studies.

\begin{tabular}{|c|c|c|c|}
\hline Author and year of Publication & {$[26]$} & {$[17]$} & [3] \\
\hline Population & $\begin{array}{l}\text { All patients undergoing } \\
\text { clean surgery }(n=660)\end{array}$ & $\begin{array}{l}\text { All patients undergoing } \\
\text { elective surgery }(n=1429)\end{array}$ & $\begin{array}{l}\text { All patients undergoing } \\
\text { clean surgery }(n=2113)\end{array}$ \\
\hline Intervention & Use of face mask & Use of face mask & Use of face mask \\
\hline Comparator & Non-use & Non-use & Non-use \\
\hline Outcomes & Postoperative wound infection & Postoperative wound infection & Postoperative wound infection \\
\hline Study Design & $\begin{array}{c}\text { RCT } \\
\text { Follow-up }=33.4 \text { days }\end{array}$ & $\begin{array}{c}\text { RCT } \\
\text { Follow-up }=\text { until after discharge }\end{array}$ & $\begin{array}{c}\text { RCT } \\
\text { Follow-up }=\text { until after discharge }\end{array}$ \\
\hline
\end{tabular}

measures of the effect of surgical facemasks in the operating room as the diagnosis of SSI comes with its unique challenges due to the heterogeneous characteristics of the surgical patient population. The available literature [3] [26] recognise the surgical patient population as a heterogeneous group with various impairments, diverse ages and varying degrees of co-morbidity. Therefore, they highlighted the difficulty in attaining the effect of intervention in the elderly and there may be distortion in results when there is co-morbidity with the disease process.

Birgand et al. [17] did not define wound infection but stressed infection serious enough to need antibiotic treatment. They examined the patients daily until discharge without stating post discharge monitoring, creating difficulty in substantiating the findings. Vincent and Edwards [3] used an outcome measure of wound infection characterised by pus or cellulitis which required wound drainage and debridement including antibiotic treatment. Record of follow up in this study was until discharge without documentation of post discharge findings. It is unclear how they were monitored post discharge, making it a weakness of the study [23].

Webster et al. [26] used the National Nosocomial Infection Surveillance method categorising SSI as superficial, deep or organ space. They followed the patients up for 6 weeks with a mean of 33.4 days for each group. The three studies did not measure level of compliance by staff with regards to appropriate use of facemasks as none reported any incidence of venting, wicking or wiggling.

\subsection{Primary Outcome}

The incidence of SSI as presented in the three studies revealed no significant statistical difference in wound infection between the masked and unmasked groups. Webster et al. [26] reported wound infection of 10.5\% in the masked group and $9.1 \%$ in the unmasked group. Brigand et al. [17] reported $4.7 \%$ wound infection in the masked and $3.5 \%$ in the unmasked group in the first trial and no wound infection in the masked and $30 \%$ wound infection in the unmasked in the second trial. Vincent and Edwards [3] reported $1.8 \%$ wound infection in the masked group and $1.4 \%$ in the unmasked. These statistics are presented in Table 7 below. 
Table 7. Summary of results and findings.

\begin{tabular}{|c|c|c|}
\hline \multicolumn{3}{|c|}{ Data Analysis (Comparison-Mask versus No mask) No. of Studies $n=3$} \\
\hline $\begin{array}{l}\text { Study or Subgroup and } \\
\text { outcome }\end{array}$ & $\begin{array}{l}\text { Main Results } \\
\text { Odds Ratio }(\mathrm{OR}=\mathrm{M}-\mathrm{H} \text { Fixed } 95 \% \text {, } \\
\text { Confidence Interval = CI) }\end{array}$ & Findings \\
\hline $\begin{array}{l}{[26]} \\
\text { Wound infection }\end{array}$ & $\begin{array}{l}\text { Mask group } 10.5 \% \\
\text { No mask group } 9.1 \% \text { No statistically significant } \\
\text { difference in SSI rate. } \\
\mathrm{OR}=1.17, \mathrm{CI}=0.70 \text { to } 1.97\end{array}$ & $\begin{array}{l}\text { Findings from the study indicate no decrease in wound } \\
\text { infection whether or not non scrubbed staff wore surgical } \\
\text { face mask during clean surgery. }\end{array}$ \\
\hline $\begin{array}{l}{[17]} \\
\text { Wound infection }\end{array}$ & $\begin{array}{l}2 \text { trials with disparate results } \\
\text { 1) Mask group } 4.7 \% \text {, No mask } 3.5 \% \text {. No statistically } \\
\text { significant difference. OR }=1.30 \mathrm{CI}=0.05 \text { to } 1.01 \text {. } \\
\text { 2) Mask group } 0 \% \text {, No mask } 30 \% \text {. Significant decrease in } \\
\text { SSI rate. }\end{array}$ & $\begin{array}{l}\text { Evidence supporting the effectiveness of face mask use } \\
\text { during clean surgery in the operating room is } \\
\text { inconclusive. It is difficult to alter current clinical } \\
\text { practice based on the findings from this study. }\end{array}$ \\
\hline $\begin{array}{l}\text { [3] } \\
\text { Wound infection }\end{array}$ & $\begin{array}{l}3 \text { trials with no statistically significant difference in SSI. } \\
\text { 1) } \mathrm{OR}=1.34, \mathrm{CI}=0.58 \text { to } 3.07 \\
\text { 2) } \mathrm{OR}=0.07, \mathrm{CI}=0.00 \text { to } 1.63 \\
\text { 3) } \mathrm{OR}=1.17, \mathrm{CI}=0.70 \text { to } 1.97\end{array}$ & $\begin{array}{l}\text { From the limited results, it is unclear whether wearing } \\
\text { surgical face mask increases or decreases SSI rate in } \\
\text { patients undergoing clean surgery. They recommend } \\
\text { further research. }\end{array}$ \\
\hline Total $(95 \% \mathrm{CI})$ & $\begin{array}{l}\text { Mask }=2055, \text { No mask } 2140 \\
\text { Weight }=100.0 \%, \text { OR }=075, \mathrm{CI}=0.56-0.99\end{array}$ & \\
\hline
\end{tabular}

Heterogeneity $\mathrm{Chi}^{\mathrm{n}}=0.02, \mathrm{df}=1(\mathrm{P}=0.90), \mathrm{1}^{\mathrm{n}}=0 \%$; Test for overall effect: $\mathrm{Z}=2.02(\mathrm{P}=0 \%)$.

\subsection{Secondary Outcome}

The secondary outcome measures specified in this systematic review were: increased care cost, longer hospitalisation, morbidity and mortality rate. Thus, researchers [48] [49] have argued that non-use of facemasks in the operating room would be an unrealistic outcome measure of achieving a reduction in SSI rate as patients with comorbidities or the elderly can get wound infection after seemingly clean surgery. Therefore, a reduction in the length of hospitalisation [17] [3], reduction in health care costs and reduced mortality rates [3] would be better outcome measures for the effectiveness of facemask use as a correlation to health care use [50]. However, none of the three studies included evaluated these outcome measures.

\subsection{Allocation}

Vincent and Edwards [3] randomised staff into two groups of masked and unmasked. Allocation was not concealed. Brigand et al. [17] randomly allocated patients on the operation list to a masked or unmasked group of the surgical team with unclear allocation concealment. Webster et al. [26] randomised participants with true allocation concealment. The unit of randomisation in all the studies was the surgical team and the patient, the unit of assessment, therefore creating error in the unit of analysis. Selection bias could not be excluded as none of the studies presented information of patient allocation to specific operation lists.

In the review, Vincent and Edwards [3] indicated that no outcome measure has proved to be the best in the effect of surgical facemask in minimising SSI. 
However, all three studies agree that the outcomes present the same results with unclear findings of the effect of surgical face mask use in preventing SSI in clean surgery in the operating room. This analysis to further investigate the outcome provided rationale for the systematic review. Meta-analysis and RCTs are methodologies of high rating within the positivist paradigm viewed as the highest in evidence rating [33]. The strength of the evidence from a meta-analysis is seen as evidence whose findings can easily be generalised to diverse populations and settings. All studies concluded that there was no significant reduction in the rate of SSI in the control groups.

Considering the strength of, and support for RCT methodology and meta-analysis, the results clearly show that the use of surgical facemasks in the operating room does not minimise the rate of SSI. However, the limitation of the RCT methodology is the setting of exclusion criteria in the process of randomisation [25]. In the attempt of controlling confounding variables, only information relating to clean surgery and RCT met the criteria for this systematic review. Therefore, the sample cannot be seen as a true representation of all surgeries done in the operating room. This could have influenced the results of the effect of surgical facemasks in reducing infection rate in the operating room.

Using a different study design, one of the studies in Birgand et al. [17] systematic review examined the effect of facemasks in a non-scrubbed group of the operating room personnel in a quasi-randomised experimental study. The limitations of the study highlighted the methodological weakness of not utilising the RCT design. However, in the view of Parahoo [25] this can be due to some practical and ethical reasons. Moreover, they stated in defence of their choice of study design, the potential to include more operating room personnel and patients in the intervention instead of just selecting a few.

In addition, the study also cited the high risk of data contamination if the sample was randomised. Notwithstanding this limitation of using a quasi-experimental design, the needs of the personnel and the patients were duely considered as a vital requirement for ethical research and to ensure the quality of the data collected [24] [51]. The results of the study revealed a reduction in the rate of surgical wound infection in the intervention group which they attributed to the robust identification criteria for patient selection [17]. On the contrary, Jni, Altman and Egger [32] acknowledged that the process of RCT sampling may have excluded patients at high risk of wound infection even after clean surgery, consequently creating bias in the result of the review [18].

Utilising a mixed method approach, one of the studies in [17] published quantitative and qualitative research findings. This study reported no significant reduction in infection rate. On the other hand, one of the articles presented results with statistical data showing a reduction in SSI rate. However, three authors [31] [32] [52] advocate the quantitative analysis, contrasting the views of Holloway and Wheeler [53] and Bunniss and Kelly [21] that the qualitative analysis provides a better understanding of the intricate challenges in healthcare practice.

Anderson [49] stated the relevance of the patient's perspective in establishing 
the quality and future direction of surgical services, suggesting that service improvement is better evaluated from the patient's perspective. Vincent and Edwards [3] obtained consent from patients as a marker of patients' willingness and improved communication between patients and staff. However, there are some similarities identified in the findings from the studies.

\subsection{Critiquing Systematic Reviews and RCTs}

A significant challenge in undertaking systematic reviews as highlighted by May and Holmes [54] is meta-analysis of the primary research studies included as they are also meta-analyses. In overcoming this challenge, they [54] advise against using data from the primary sources more than once to avoid duplicating results, thus analysing each source and subsequently combining results from the individual studies. Jni, Altman and Egger [32] view the RCT as the gold standard in providing evidence of effectiveness and the highest in evidence rating. However, this quantitative methodology can stand the risk of skipping some of the most vital information [23]. Therefore, Carey [55] and Engward [51] advised the inclusion of qualitative data. However, only studies with quantitative design met the inclusion criteria for this review.

The literature highlighted some key factors for analysis. The diagnosis of a surgical wound infection comes with its unique challenges. For instance, a considerable number of patients like the elderly and the immuno-compromised with co-morbidities are a heterogeneous group with diverse problems that make it complex to measure outcomes of care as they do not always manifest the most important signs of infection. An overview of the results of the literature search and findings has been presented and will be discussed in the next section.

\section{Discussion}

Although facemasks are widely used and adjudged good practice, it is still a topic under-researched. Evidence has shown that current national and international policies on the use of facemasks are based on laboratory studies using settle plates to estimate potential surgical field contamination without direct investigation of surgical wounds. This type of indirect evidence is questionable in relevance to clinical practice. One of the three studies that met the inclusion criteria presented weak evidence. Two studies presented unclear allocation concealment, an essential part of RCT [22].

The results of Webster et al. [26] and Birgand et al. [17] may have several methodological bias. Birgand et al. [17] did not specify the criteria used in detecting SSI. As highlighted by Mangram et al. [47], failure to state the criteria used objectively to determine the presence of SSI can create bias in reporting SSI rate in a study. One of the studies in Birgand et al. [17] discontinued the trial when wound infection was detected in the intervention group, therefore, creating bias in the findings. The follow up in this study was till discharge and after discharge in Webster et al. [26]. The actual follow up time may have varied according to 
the category of surgery, causing an eventual under estimation of the SSI rate [48]. Webster et al. [26] were more in line with international guidelines as they followed up the patients for 33.4 days.

Under or over estimation of the effectiveness of facemask use could have resulted from the lack of blinding and allocation concealment in the studies of Birgand et al. [17] and Vincent and Edwards [3]. Testing publication bias was impossible as a result of the limited number of studies that met the inclusion criteria for this study. This was due to the surprisingly limited number of RCTs on this topic. Critical appraisal of the validity of the included studies depended on adequate report on the trials, which was limited in some cases. Therefore, it would be erroneous to conclude that the method of rigour was inadequate with such limited information. However, attempts at obtaining more information from experts in the field and investigators of the studies yielded no positive response.

Additionally, judgement of the effectiveness of facemask must consider all variables in association with SSI. It is impossible to determine the impact of facemask use on SSI rate after clean surgery with small studies like Birgand et al. [17]. The result from this study cannot be generalised to other categories of surgery as the review was limited to clean surgery only. However, the consideration for reviewing only clean surgery was drawn from this researcher's opinion that facemask use in the prevention of SSI is less likely to have impact in an already contaminated wound.

Although the review included all patients undergoing clean surgery, none of the studies was specific on prosthetic implant surgery. Therefore, the application of the findings to this category of surgery will be limited. Webster et al. [26] randomised non scrubbed staff alone, making it difficult to distinguish between the role of scrubbed and un-scrubbed surgical team members in any SSI resulting from the trial. Furthermore, Vincent and Edwards [3] argued that the position of non-scrubbed staff in contaminating the surgical site is less likely. However, as all studies included in the review were operating room based surgeries, application of the results to invasive procedures performed in clinical areas outside the operating room is less likely.

This study only examined the effect of facemask use on surgical patients as the potential for surgical team protection from the patient was not included in the review. Although Birgand et al. [17] advocated the use of facemask, the relative small size and presence of wound infection in some of the patients may have created bias in the results in favour of facemask use. Conversely, the other two studies [3] [26] with bigger trials and more rigorous design presented no variation in infection rate.

\section{Limitations}

The systematic review presented a number of limitations. The heterogeneous nature of the surgical patient population, inappropriate use of facemasks by 
personnel, the study design and findings created difficulty in presenting evidence-based conclusions. The fact that this systematic review was undertaken by a single researcher presented a unilateral decision in the selection of studies, a potential source of bias as general conclusions cannot be drawn without the standard appraisal of two or more researchers. However, the use of a research protocol framework made guidelines available for limiting bias. None of the reviewed studies included Nigerian stakeholders and there was none undertaken in Nigeria. Therefore, the findings and recommendations may be limited in application to Nigerian health settings.

\section{Conclusion}

The results from the studies included in this systematic review presented the same findings of unclear evidence supporting the use of surgical facemask in the operating room in preventing SSI. All the authors are of the strong opinion that more research is needed to end the controversy on their effectiveness. National and international guidelines recommend the continuous use of facemask in the operating room despite the existing controversy on the practice. However, as no other studies were identified on this topic, it will be unwise to draw decisive conclusions from the limited number of studies that met the inclusion criteria for this review on the effectiveness of facemask in minimising SSI after clean surgery.

\section{Recommendation for Practice}

It is not clear from the limited results whether facemask use minimises or increases SSI rate after clean surgery in the operating room. Although it is clear from the included studies that there is no significant evidence to support facemask use in the operating room in preventing SSI, however, it will be difficult to alter current clinical practice. This reviewer supports the continued use of surgical facemask in the operating room to maintain theatre discipline. Therefore, as there is no current empirical evidence against the use of facemask in the operating room, it is recommended that local operating departments follow international guidelines in formulating policies for promoting their continuous use.

Moreover, it was clear from the studies that surgical facemask is used inappropriately and practice is inconsistent. It is therefore recommended that training and retraining of operating room personnel be organised periodically through in-service education programmes and workshops on the appropriate use of facemask in the operating room. It is also recommended that evidence-based practice be embraced in providing operating room personnel with the necessary skills and knowledge in appraising research evidence and understanding scientific papers as experience is needed to interpret the specific vocabulary. It is only by continuous learning that the surgical team members can be confident in incorporating research evidence into the day-to-day individualised care of the surgical patient to improve overall patient outcome. 


\section{Recommendations for Further Research}

From the limited results and apparent paucity of evidence, it is clear that there is a need for further comprehensive research on this topic which should include the following:

- A standard guideline like the CONSORT statement for reporting future trials [56].

- Trials should differentiate between scrubbed and non-scrubbed staff, appropriate use of facemask and be large enough to recognise the presence of a difference in infection rate that will be of clinical importance.

- Use of a standard outcome measure of SSI.

- Randomisation should be with allocation concealment.

- Follow-up should be standardised to international guideline of 30days.

- Future studies should include evaluation of the secondary outcome measures of the financial and social effects and the mortality rate of SSI.

\section{Dissemination}

This systematic review is intended for use in hospital operating theatres to inform decision-making on appropriate use of facemasks in the operating room and to present the evidence-base supporting the practice in preventing or reducing SSI. The study aimed to prove whether facemask use is a mere routine ritual as it is yet unclear whether the use has any impact in reducing SSI after clean surgery in the operating room.

\section{Conflicts of Interest}

The author declares no conflicts of interest regarding the publication of this paper.

\section{References}

[1] Phillips, N. (2012) Berry and Kohn's: Operating Room Technique. 12th Edition, Mosby, Somerset.

[2] Canadian Agency for Drugs and Technologies in Health (2014) Use of Surgical Face Masks in the Operating Room: A Review of the Clinical Effectiveness and Guidelines.

[3] Vincent, M. and Edwards, P. (2016) Disposable Surgical Face Masks for Preventing Surgical Wound Infection in Clean Surgery. Cochrane Database of Systematic Reviews, 4, Article No. CD002929. https://doi.org/10.1002/14651858.CD002929.pub3

[4] Petticrew, M. and Roberts, H. (2006) Systematic Reviews in the Social Sciences: A Practical Guide. Blackwell Publishing, Oxford.

[5] Garner, J.S. and Hospital Infection Control Practices Advisory Committee (1996) Guidelines for Isolation Precautions in Hospital. Infection Control and Hospital Epidemiology, 17, 53-80. https://doi.org/10.2307/30142367

[6] Belkin, N.L. (1997) The Evolution of the Surgical Mask: Filtering Efficiency versus Effectiveness. Infection Control and Hospital Epidemiology, 18, 49-57. https://doi.org/10.2307/30141964

[7] Spruce, L. (2014) Back to Basics: Preventing Surgical Site Infections. AORN Journal, 
99, 600-611. https://doi.org/10.1016/j.aorn.2014.02.002

[8] Association of Operating Room Nurses (2014) Standards, Regulations Practice and Guidelines. Association of Operating Room Nurses, Denver.

[9] Borlang, G. (2016) Infection Control and Prevention: Standard Precautions-Infection Control Principles and Practice for Local Health Agencies.

[10] European Centre for Disease Prevention and Control (2012) Annual Epidemiological Report on Communicable Diseases in Europe.

[11] Plowman, R., Graves, N. and Griffin, M. (2000) The Socioeconomic Burden of Hospital Acquired Infection. Eurosurveillance, 5, Article ID: 00004. https://doi.org/10.2807/esm.05.04.00004-en

[12] Office of National Statistics (2011) MRSA SSI Surgical Site Infections on Deaths Involving MRSA.

[13] Lipp, A. and Edwards, P. (2015) Disposable Face Masks: A Systematic Review. Canadian Operating Room Nursing Journal, 23, 20-21, 24-25, 33-38.

[14] World Health Organisation (2014) Millennium Development Goals (MDGs).

[15] National Institute for Health and Care Excellence (2015) Prevention and Treatment of Surgical Site Infection.

[16] Centre for Disease Control and Prevention (2017) Guidelines for the Prevention of Surgical Site Infection. JAMA Surgery, 152, 784-791.

http://dx.doi.org/10.1001/jamasurg.2017.0904

[17] Birgand, G., Azevedo, C., Toupet, G., Pissard-Gibollet, R., Grandbastien, B., Fleury, E. and Lucet, J. (2014) Attitudes, Risk of Infection and Behaviours in the Operating Room (the ARIBO Project): A Prospective, Cross-Sectional Study. BMJ Open, 4, e004274. https://doi.org/10.1136/bmjopen-2013-004274

[18] Bettany-Saltikov, J. (2014) How to Do a Systematic Literature Review in Nursing: A Step by Step Guide. Open University Press, Maidenhead.

[19] Jolley, J. (2010) Introducing Research and Evidence-Based Practice for Nurses. Pearson, Harlow.

[20] Weaver, K. and Olson, J.K. (2006) Understanding Paradigms Used for Nursing Research. Journal of Advanced Nursing, 53, 459-469. https://doi.org/10.1111/j.1365-2648.2006.03740.x

[21] Bunniss, S. and Kelly, D.R. (2010) Research Paradigms in Medical Education Research. Medical Education, 44, 358-366. https://doi.org/10.1111/j.1365-2923.2009.03611.x

[22] Jadad, A. and Enkins, M.W. (2010) Randomised Controlled Trials: Questions, Answers and Musings. 2nd Edition, BMJ Books, Malden.

[23] Bryman, A. (2012) Social Research Methods. 4th Edition, Oxford University Press, Oxford.

[24] LoBiondo-Wood, G. and Haber, J. (2014) Nursing Research: Methods and Critical Appraisal for Evidence-Based Practice. Journal of Nursing Regulation, 5, 60. https://doi.org/10.1016/S2155-8256(15)30102-2

[25] Parahoo, K. (2014) Nursing Research: Principles, Process and Issues. 3rd Edition, Palgrave MacMillan Press Ltd., Basingstoke.

[26] Webster, J., Croger, S., Lister, C., Doidge, M., Terry, M.J. and Jones, I. (2010) Use of Surgical Face Masks by Non-Scrubbed Operating Room Staff: A Randomised Controlled Trial. ANZ Journal of Surgery, 80, 169-173. https://doi.org/10.1111/j.1445-2197.2009.05200.x 
[27] National Institutes of Health Research Involving Human Subjects (2016) Ethical Guidelines and Regulations.

[28] David, B. and Resnik, J.D. (2015) What Is Ethics in Research and Why Is It Important? National Institute of Environmental Health Sciences. http://www.niehs.nih.gov/research/resources/bioethics/whatis

[29] Brouwers, M.C., et al. (2010) AGREE II: Advancing Guidelines Development, Reporting and Evaluation in Healthcare. Canadian Medical Association Journal, 182, E839-E842. https://doi.org/10.1503/cmaj.090449

[30] Moule, P. and Goodman, M. (2014) Nursing Research. 2nd Edition, Sage, Los Angeles.

[31] Hek, G. and Moule, P. (2014) Making Sense of Research: An Introduction for Health and Social Care Practitioners. 4th Edition, Sage, London.

[32] Jni, P., Altman, D.G. and Egger, M. (2013) Systematic Reviews in Health Care: Assessing the Quality of Controlled Clinical Trials. British Medical Journal, 323, 42-46.

[33] Fain, J. (2013) Reading and Understanding and Applying Nursing Research. F.A. Davis Company, Philadelphia.

[34] Review Manager (2014) The Cochrane Collaboration. The Nordic Cochrane Centre, Copenhagen.

[35] Alwitry, A. (2013) Effect of Surgical Face Masks: Microbial Contamination on Used Surgical Face Masks among Personnel. Oman Medical Journal, 29, 346-350.

[36] McGovern, P.D. (2013) The Influence of Surgical Hoods and Togas on Airborne Particle Concentration at the Surgical Site: An Experimental Study. Journal of Orthopaedic Science, 18, 1027-1030. https://doi.org/10.1007/s00776-013-0445-7

[37] Salassa, T.E. (2014) Surgical Attire and the Operating Room: Role in Infection Prevention. The Journal of Bone and Joint Surgery, 96, 1485-1492. https://doi.org/10.2106/JBJS.M.01133

[38] Hubble, M.J., Weale, A.E., Perez, J.V., Bowker, K.E., MacGowan, A.P. and Bannisster, G.C. (1996) Clothing in Laminar-Flow Operating Theatres. Journal of Hospital Infection, 32, 1-7. https://doi.org/10.1016/S0195-6701(96)90159-0

[39] Mitchell, N.J. and Hunt, S. (1991) Surgical Face Masks in Modern Operating Rooms-A Costly and Unnecessary Ritual? Journal of Hospital Infection, 18, 239-242. https://doi.org/10.1016/0195-6701(91)90148-2

[40] Chamberlain, G. (2014) Trial of the Use of Surgical Face Masks in the Gynaecological Theatre. Annals of the Royal College of Surgeons of England, 66, 432-433.

[41] Bahli, Z.M. (2009) Does Evidence Based Medicine Support the Effectiveness of Surgical Face Masks in Preventing Postoperative Wound Infection in Elective Surgery? Journal of Ayub Medical College, Abbottabad, 21, 166-170. http://www.ayubmed.edu.pk/JAMC/PAST/21-2/Zahid.pdf

[42] Belkin, N.L. (2011) The Evolution of the Surgical Mask: Filtering Efficiency versus Effectiveness. Infection Control and Hospital Epidemiology, 18, 49-57. https://doi.org/10.2307/30141964

[43] Romney, M.G. (2001) Surgical Face Masks in the Operating Theatre: Re-Examining the Evidence. Journal of Hospital Infection, 47, 251-256. https://doi.org/10.1053/jhin.2000.0912

[44] Ha'eri, G.B. (2012) The Efficacy of Standard Surgical Face Masks: An Investigation Using “Tracer Particles”. Clinical Orthopaedics and Related Research, 148, 160-162. https://doi.org/10.1097/00003086-198005000-00024

[45] Berger, S.A., Kramer, M., Nagar, H., Finkelstein, A., Frimmerman, A. and Miller, 
H.I. (1993) Effect of Surgical Face Mask Position on Bacterial Contamination of the Operating Field. Journal of Hospital Infection, 23, 51-54. https://doi.org/10.1016/0195-6701(93)90130-R

[46] Sjol, A. (2015) Is Use of Surgical Caps and Masks Obsolete during Percutaneous Heart Catheterisation? Ugeskrift for Læger, 164, 1673-1675.

[47] Mangram, A., Horan, T., Pearson, M., Silver, L., Jarvis, W., et al. (1999) Guideline for Prevention of Surgical Site Infection. Infection Control and Hospital Epidemiology, 20, 247-278. https://doi.org/10.1086/501620

[48] Anderson, D.J. (2015) Surgical Site Infection. Infectious Disease Clinics of North America, 25, 135-153. https://doi.org/10.1016/j.idc.2010.11.004

[49] Kulayat, M.N. and Dayton, M. (2012) Textbook of Surgery. 19th Edition, Elsevier Saunders, Philadelphia.

[50] Looman, W.M., Fabbricotti, I.N. and Huijsman, R. (2014) The Short-Term Effects of an Integrated Care Model for the Frail and Elderly on Health, Quality of Life, Health Care Use and Satisfaction with Care. International Journal of Integrated Care, 14, e034.

[51] Engward, H. (2013) Understanding Grounded Theory. Nursing Standard, 28, 37-41.

[52] Khan, K.S., Kunz, R., Kleijnen, J. and Antes, G. (2011) Systematic Reviews to Support Evidence Based Medicine: How to Review and Apply Findings to Healthcare Research. 2nd Edition, CRC Press, London.

https://doi.org/10.1201/b13411

[53] Holloway, I. and Wheeler, S. (2010) Qualitative Research in Nursing and Health Care. 3rd Edition, Wiley-Blackwell, Chichester.

[54] May, A. and Holmes, S. (2015) Introduction to Nursing Research: Developing Research Awareness. [e-book] CRC Press. Available through Anglia Ruskin University Library website.

[55] Carey, M. (2013) The Social work Dissertation: Using Small-Scale Qualitative Methodology. 2nd Edition, McGraw Hill Open University Press, Maidenhead.

[56] Schulz, K.F. (2010) CONSORT 2010 Statement: Updated Guidelines for Reporting Parallel Group Randomised Trials. Annals of Internal Medicine, 152, 726-732. https://doi.org/10.7326/0003-4819-152-11-201006010-00232 


\section{Appendix 1: Search Strategy}

\begin{tabular}{cclcc}
\hline $\begin{array}{c}\text { Database } \\
2001-2016\end{array}$ & Search Date & \multicolumn{1}{c}{ Keywords } & $\begin{array}{c}\text { No of Hits } \\
\text { retrieved }\end{array}$ & $\begin{array}{c}\text { No of Hits } \\
\text { discarded }\end{array}$ \\
\hline $\begin{array}{c}\text { Google } \\
\text { Scholar }\end{array}$ & $\begin{array}{c}\text { February } \\
2017\end{array}$ & $\begin{array}{l}\text { "surgical facemask in operating room in clean } \\
\text { surgery" }\end{array}$ & 32 & 29 \\
& & & \\
"facemask in operating room" "surgical* AND \\
BNI
\end{tabular}

\section{Appendix 2: Definition of Keywords}

Operating Room: A facility within a hospital where surgical operations are carried out in an Aseptic environment.

Operating Theatre: A term also used for a place where surgery takes place in a hospital.

Surgical Facemask: A rectangular-shaped piece of fabric or polypropylene fibre worn by medical Personnel over the nose and mouth, secured by straps tied at the back of the Head during surgical procedures intended to protect the wearer against splashes or spatters of blood or body fluid and to prevent contamination of the patient's surgical wound.

Clean Surgery: An incision in which no inflammation is encountered in a surgical procedure, without a break in sterile technique, and during which the respiratory, alimentary and genitourinary tracts are not entered.

Surgical Site Infection: An infection that occurs in the incision created by an invasive surgical Procedure. 